\title{
Sistema experto para regular tensión en redes de distribución
}

ELECTRIC AND ELECTRONIC ENGINEERING

\section{Expert system for regulating voltage in Distribution Network}

\author{
Alfredo Cervera*, Gladys Caicedo-Delgado**§ \\ *Centro de Supervisión y Maniobras, Gerencia de Gestión de Energía, Empresa de Energía del Pacifico ESP, \\ Yumbo- Colombia \\ **Escuela de Ingeniería Eléctrica y Electrónica, Universidad del Valle Cali, Colombia \\ §acervera@epsa.com.co,glacadel@univalle.edu.co
}

(Recibido: enero 24 de 2014 - Aceptado: octubre 16 de 2014)

\begin{abstract}
Resumen
Este artículo presenta una aplicación basada en la experiencia operativa de 22 años para regular tensión en el Sistema de Distribución de la Empresa de energía del Pacífico (EPSA). Por lo tanto, se presenta un Sistema Experto (SE) basado en reglas heurísticas que utilizan los operadores y que han sido probadas experimentalmente. Las maniobras ejecutadas restablecen los niveles de tensión en el $95 \%$ de los nodos, no sobrepasan los límites térmicos de los diferentes elementos del sistema, ni los limites operativos de seguridad del sistema. Este SE desarrollado en MATLAB utilizó 8 reglas heurísticas para identificar el tipo de problema de regulación y para definir los recursos que compensaran los reactivos para mejorar los niveles de tensión. La validación se realizó sobre el modelo del SEP implementado en el software Digsilent. El aporte de este trabajo es que se integra en la solución, criterios de regulación considerando la operación conjunta del sistema de transmisión y distribución, lo cual es más cercano a la realidad.
\end{abstract}

Palabras claves: Redes de distribución, reglas heurísticas, regulación de tensión, sistema experto.

\begin{abstract}
This paper presents an application based on the operational experience of 22 years to regulate voltage in the distribution system of Pacific Energy Company (EPSA). Therefore, an expert system (SE) based on heuristic rules used by operators and have been experimentally tested is presented. The maneuvers executed reset voltage levels in $95 \%$ of the nodes, do not exceed the thermal limits of the various elements of the system, or system operating limits. This SE developed in MATLAB 8 heuristic rules used to identify the type of regulation problem and to define the resources to offset the reactive to improve voltage levels. Validation was performed on the model of SEP implemented in DIgSILENT software. The contribution of this work is that it is integrated into the solution, regulatory criteria considering the joint operation of transmission and distribution system, which is closer to reality.
\end{abstract}

Keywords: Distribution networks, expert system, heuristic rules, voltage regulation. 


\section{Introducción}

La regulación de tensión confiable en un Sistema Eléctrico de Potencia (SEP), es uno de los requerimientos más importantes para brindar una alta calidad de servicio al usuario. Puesto que el perfil de la tensión varía constantemente debido a fluctuaciones de: carga, cambios de generación y/o cambios topológicos por fallas o mantenimientos, éste debe ser controlado apropiadamente operando los recursos disponibles de potencia reactiva que permitan mantener la estabilidad de la tensión y la calidad de la energía del sistema.

Mantener los niveles de tensión adecuados durante la operación normal y después de una perturbación se relaciona con el balance de potencia reactiva entre la generación, la carga y las pérdidas del sistema. Si se genera un desbalance de potencia reactiva en el sistema, es necesario compensarla para evitar la variación de los voltajes en los nodos, para ello se utilizan elementos compensadores de reactivos con el propósito de balancear el sistema y mantener la calidad de la tensión (Kundur., 1994).

Con este objetivo, se han diseñado e implementado estrategias de regulación de tensión en sistemas de distribución como los sistemas de control jerárquico distribuido multiagente (Geao et al., 2005). Este esquema ha sido implementado en el Sistema Eléctrico de Nueva Inglaterra, resultando muy eficaz en el manejo del perfil de tensión de todo el sistema de potencia en operación normal y en contingencia. También se realizó el control de tensión y optimización de potencia reactiva en el sistema de distribución de Yunlin Taiwán, el cual muestra la eficacia de un método de programación dinámica para el control de potencia reactiva y tensión con cambiadores de taps y condensadores en una subestación de distribución (Ruey et al., 2003).

Además se han utilizado técnicas de inteligencia artificial para regular perfiles de tensión en redes interconectadas de distribución, tal como los SE que han resultado eficaces en redes bajo diferentes condiciones de carga, sus resultados obtenidos demuestran su robustez y su capacidad para regular tensiones en los nodos y reducir al mínimo los movimientos de control (Azmy., 2007). El método aplicado para regular tensión en el Sistema Eléctrico de la provincia de Alberta en Canadá mediante un SE, ha permitido obtener como resultado un perfil unificado de tensiones, despacho óptimo de potencia reactiva. En este sentido el SE ha resultado bastante apto, también ha mostrado gran flexibilidad para el tratamiento de problemas de difícil formulación (Vaschetti et al., 2012).

La regulación de tensión en el sistema de distribución del Valle del Cauca desde el punto de vista operativo, ha sido un problema complejo por los siguientes motivos: El tamaño del sistema de distribución es grande, disperso geográficamente, la característica de la carga es muy variada, existe diversidad y ubicación de equipos de control de potencia reactiva, no se tiene autonomía en el manejo de los recursos en el nivel de tensión del Sistema de Interconectado Nacional (SIN), es decir a nivel de $550 \mathrm{KV}$ y $220 \mathrm{KV}$.

EPSA ha dividido su sistema de distribución en cinco zonas, las cuales corresponden a partes del sistema cuyos nodos están cercanos geográficamente y el comportamiento de su variación de voltaje es similar. Para implementar el SE en este trabajo, algunas de estas zonas geográficas se agruparon en áreas. Para la definición de las áreas, se evaluó la información histórica del comportamiento de los voltajes en nodos del Sistema de Transmisión Regional (STR) y del Sistema de Distribución Local (SDL), se agruparon los nodos de diferentes zonas que presentaban variaciones similares en una misma área. Es decir, la organización por áreas permitió agrupar un mayor número de nodos con los mismos problemas de desviaciones, facilitar la identificación de la severidad del problema de regulación e identificar los equipos existentes más cercanos a los nodos desviados para compensar los reactivos. La zona de Buenaventura por ser un sistema aislado y estar conectada SEP a través de una sola línea, se consideró como una sola área. En la Figura 1 se ilustra la organización del SEP por zonas y por áreas.

A través del sistema de Supervisión, Control y Adquisición de Datos de las variables eléctricas (SCADA), se midieron las tensiones en los todos los nodos de referencia y nodos indicadores del 


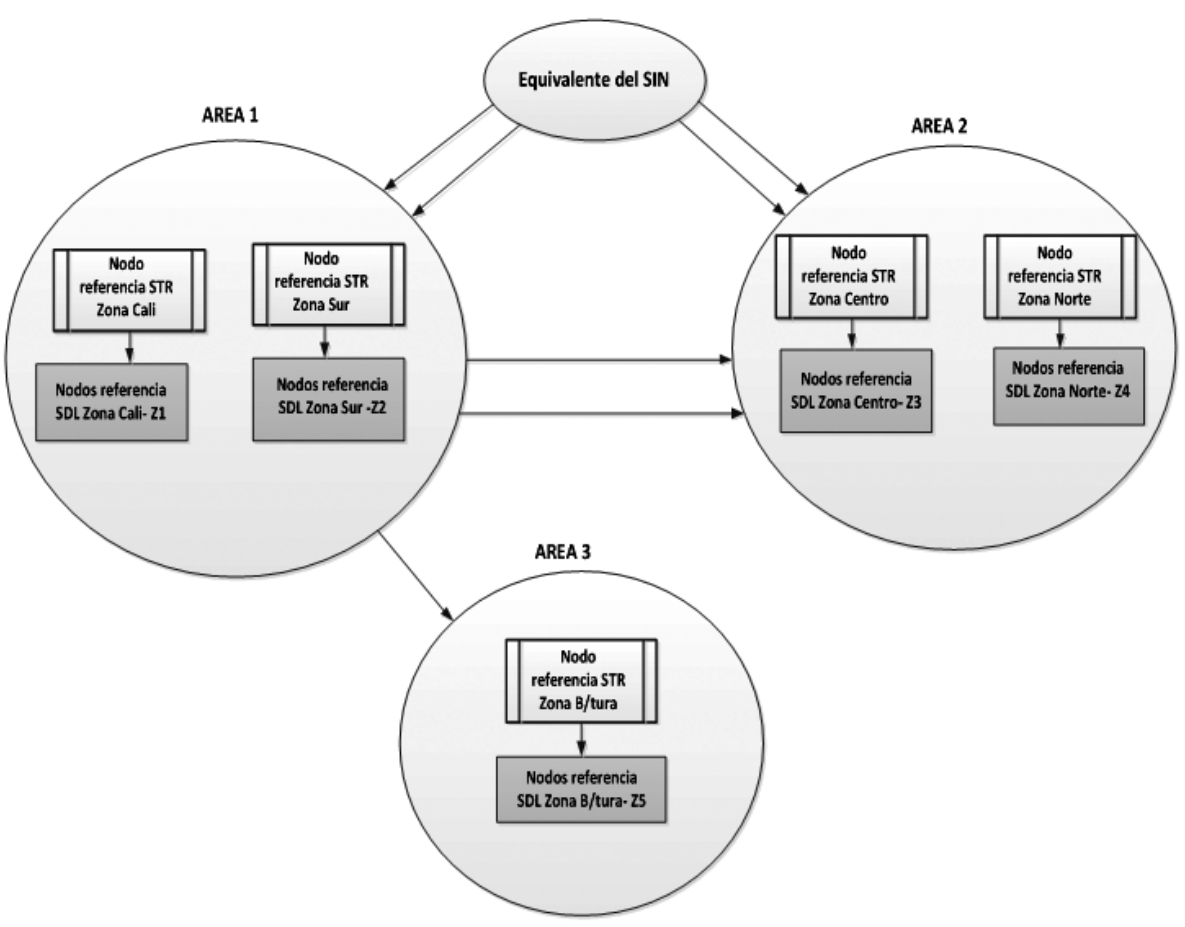

Figura 1. Estructura de validación del SE propuesto para regular tensión

SEP en estudio. Los nodos de referencia son aquellos nodos del SEP que tienen influencia en la tensión de otros nodos aguas abajo, y que su nivel de tensión se puede variar porque cuenta con recursos instalados para variar sus reactivos: condensador (en algunos casos) y taps del transformador. Se definieron varios de estos nodos de referencia por zona en el SDL a nivel de $34,5 \mathrm{KV}$ y $13,2 \mathrm{KV}$ y se definió uno por zona en el STR a nivel de $115 \mathrm{KV}$.

Los nodos indicadores, son aquellos nodos del STR que su tensión varia cuando se presenta una variación de la tensión en los nodos del SIN a nivel de $500 \mathrm{KV}$ y $200 \mathrm{KV}$. Además, en estos nodos indicadores su tensión varía con menos sensibilidad que la tensión en los nodos de referencia. En el SE se seleccionó un solo nodo indicador por zona en el STR a nivel de $115 \mathrm{KV}$. En la Tabla 1, se presentan los nodos de referencia e indicadores seleccionados por áreas y zonas en el SEP en estudio.

Una vez leídos los datos de las tensiones, se calculan las desviaciones de todos los nodos de referencia y nodos indicadores del SEP. Cuando estos nodos tienen tensiones por fuera de los rangos operativos de acuerdo a su cantidad y su ubicación por zonas y áreas, se identifica por medio de reglas heurísticas el tipo de problema de regulación de tensión a resolver. A continuación se describe cada una de esta Reglas Heurísticas (RE) para identificar cada uno de los tipos de problema: local (si existe un solo nodo de referencia del SDL desviado, en cualquier zona), zonal (si existen varios nodos de referencia del SDL desviados en una misma zona), área (si existen varios nodos de referencia del SDL desviados de diferentes zonas de la misma área) o general (si existen varios nodos de referencia del SDL desviados en diferentes áreas. Cuando todos los nodos indicadores del STR están desviados, se confirma el problema regulación de tensión es de tipo general).

De acuerdo a la identificación del tipo de problema de regulación, su ubicación y severidad. SE con base a otras RE, prioriza y define las maniobras a realizar sobre los equipos de control de reactivos más cercanos a los nodos de referencia en el SDL y STR desviados.

\section{Metodología}

El desarrollo de esta investigación se realizó en cuatro etapas: La primera realiza la descripción del 
Tabla 1. Nodos de referencia e indicadores en STR y SDL

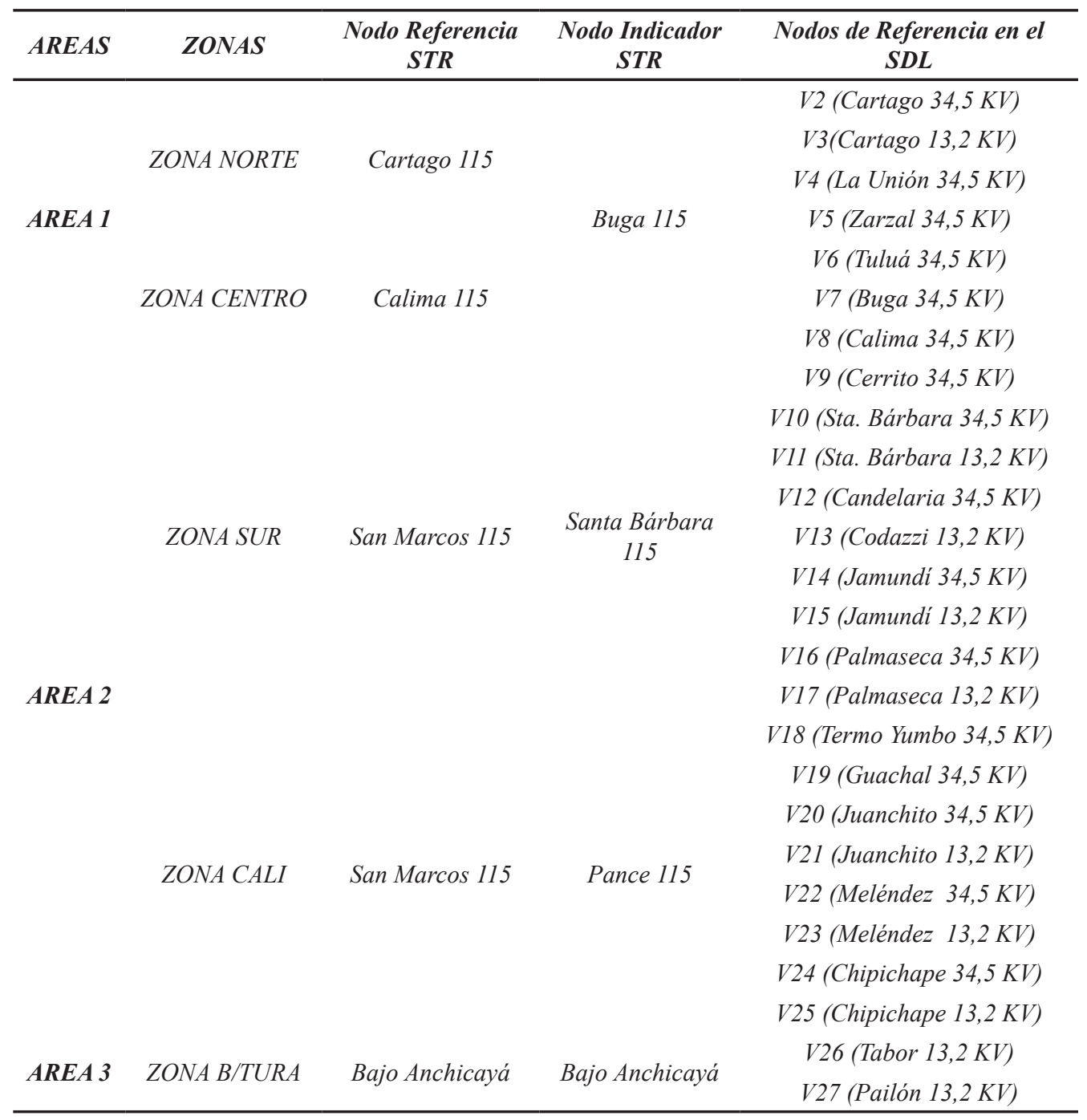

SEP, la segunda muestra el diagrama de bloques del SE) propuesto: sus señales de entrada, la descripción funcional de cada uno de los bloques y sus señales de salida. En la tercera etapa se describe la validación del sistema experto y se ilustra un caso de simulación que se utilizó para validarlo. A continuación se describe en detalle cada una de las etapas mencionadas:

\subsection{Descripción del sistema de potencia a regular}

En la figura 2 se ilustra la estructura del SEP del Valle del Cauca y su ubicación de los recursos de potencia reactiva disponibles para la regulación de tensión. Este sistema está constituido por varios niveles de tensión: los niveles superiores denominados de transmisión $220 \mathrm{KV}$ y $500 \mathrm{KV}$, los niveles intermedios denominados de subtransmisión $115 \mathrm{KV}$ y los niveles inferiores denominados de distribución 34,5 KV y 13.2 KV. Debido a esta estructura jerárquica, para una obtener una adecuada regulación de tensión en distribución, se requiere verificar el estado del nivel de tensión en la red de transmisión y subtransmisión. Dado que EPSA en este SEP es el único operador de red responsable de mantener los niveles de tensión en los nodos de distribución y subtransmisión, se propuso con el sistema experto regular los niveles de tensión en los nodos 


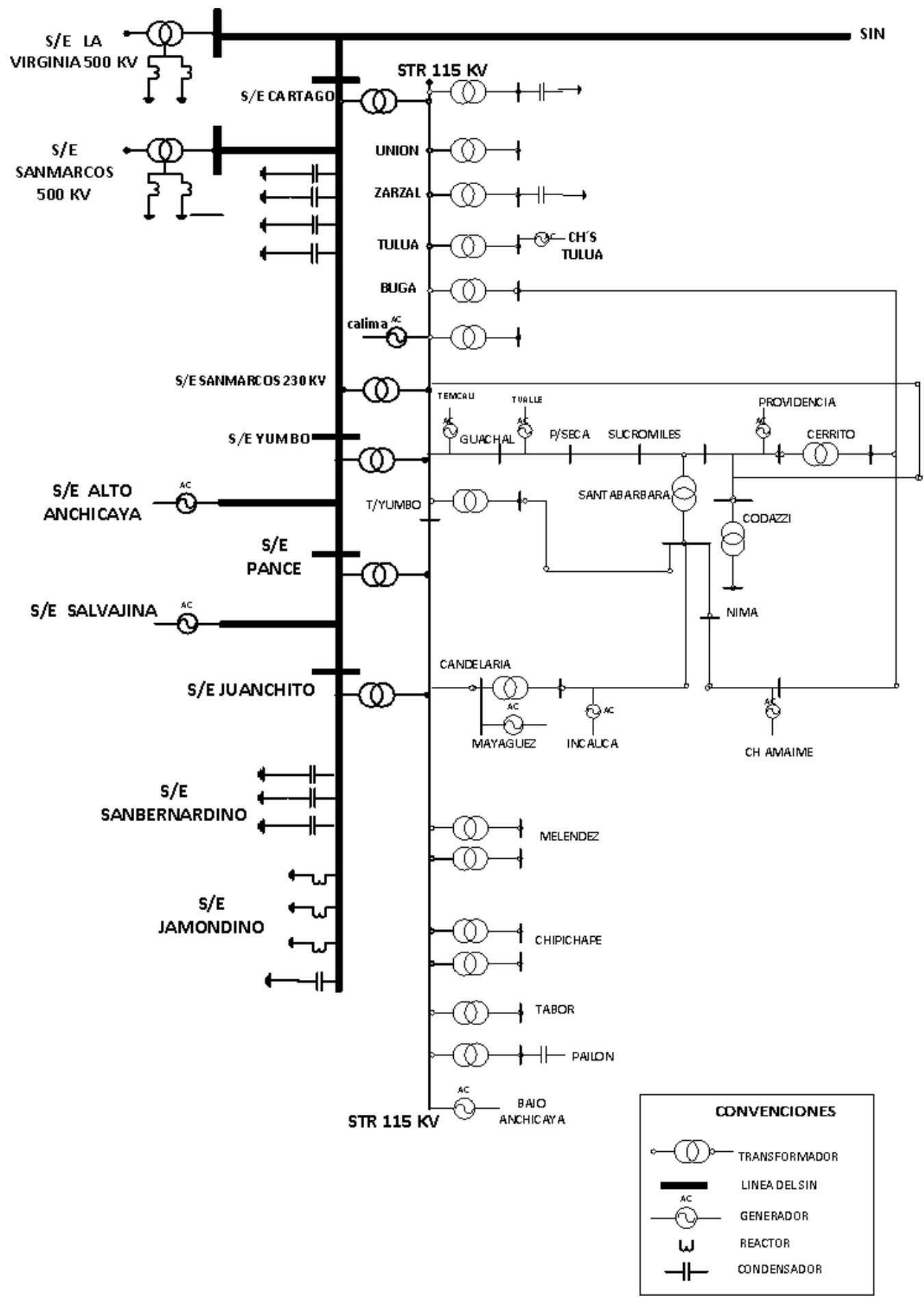

Figura 2. SEP del Valle del Cauca y su ubicación de los recursos de potencia reactiva disponibles 


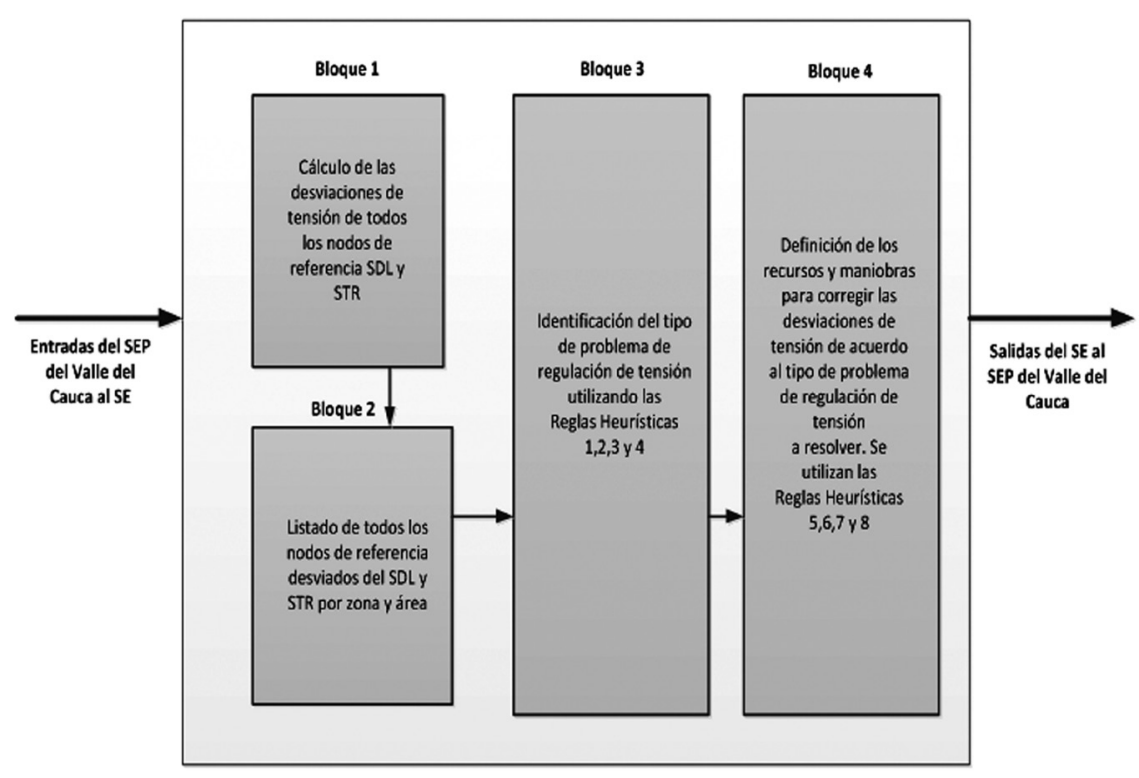

Figura 3. Diagrama de bloques del SE para regular tensión

de distribución, considerando los valores de las tensiones en los nodos de subtransmisión.

Este sistema de transmisión se caracteriza por tener líneas largas en la Región Norte del Valle (Yumbo, San Marcos, Cartago) y líneas cortas en el resto de la región. La generación tiene componente hidráulico en $220 \mathrm{KV}$ con generación en: Alto Anchicayá y Salvajina, y en $115 \mathrm{KV}$ con generación en: Bajo Anchicayá, Calima, y las térmicas de Termoemcali y TermoValle. Adicionalmente, cuenta con la conexión de los cogeneradores en $115 \mathrm{KV}$ de los ingenios Mayagüez y Providencia y por $34.5 \mathrm{KV}$ del Ingenio de Cauca. También cuenta con los sgtes recursos para el control de tensiones: 4 bancos de compensación capacitiva de 72 MVARs en San Marcos a 220 kV, 2 bancos de compensación reactiva de 50 MVARs en el terciario del transformador de San Marcos 500/230/34.5 KV, y 2 bancos de compensación reactiva de 50 MVARs en el terciario del transformador de La Virginia 500/230/34.5 KV.

El Valle del Cauca tiene 8 puntos de interconexión que constituyen puntos frontera con otras áreas, la circulación de potencia a través de estos puntos impacta significativamente el comportamiento de la tensión en la red de distribución.

\subsection{Estructura del SE propuesto}

La figura 3 muestra los diagramas de bloques del SE propuesto. A continuación se describen: sus señales de entrada del SEP que son tomadas a través del SCADA, la función de cada uno de los bloques y sus señales de salida.

\subsubsection{Señales de entrada al SE}

A continuación se presentan las señales de entrada al SE:

Variables para determinar el estado operativo: las tensiones actuales de los nodos de referencia en el SDL y STR, las tensiones actuales de los nodos indicadores en el STR, las potencias activas actuales de los transformadores y las potencias reactivas actuales de los generadores y cogeneradores.

Variables para determinar la disponibilidad de los recursos: limites operativos de las tensiones en cada nodo de referencia del SDL y STR y cada nodo indicador del STR, limites operativos de potencia reactiva de generadores y cogeneradores, límites operativos de potencia activa de los transformadores, límites y posiciones actuales de cambiadores de taps de los transformadores, estado de la posición del interruptor de condensadores y reactores. 
Todas estas señales de entrada y recursos de reactivos están identificados por zonas y por áreas.

\subsubsection{Función de cada uno de los bloques del SE:}

El SE ilustrado en la Figura 3 contiene en cuatro bloques, los cuales de describen a continuación.

Bloque 1: Este bloque tiene como función, realizar el cálculo de las desviaciones de tensión de los nodos de referencia en el SDL y STR. Estas desviaciones se determinan realizando la diferencia de los límites operativos y las mediciones de tensión de cada nodo. El resultado determina si esta desviación es una sobretensión o una subtensión. Dado que se trata de un SEP jerárquico, todos los nodos presentaran desviaciones por sobretensión o por subtensión.

Bloque 2: Este bloque tiene como función contabilizar el número de nodos de referencia del SDL y STR y el número de nodos indicadores desviados por zonas y por áreas.

Bloque 3: La función de este bloque es identificar el tipo de problema de regulación de tensión: local, zonal, área y general. Para ello se utilizan una regla heurística para identificar cada uno de estos problemas. Estas reglas heurísticas son:

Regla heurística para identificar el problema de regulación local: Si en una zona i, existe un solo nodo de referencia del SDL desviado, el problema de regulación es local.

Regla heurística para identificar el problema de regulación de zona: Si en una zona i, existe más de un nodo de referencia del SDL desviado, el problema de regulación es zonal.

Regla heurística para identificar el problema de regulación de área: Si en una misma área $i$, existe por lo menos un nodo de referencia del SDL desviado en varias de sus zonas, el problema de regulación es de área.

Regla heurística para identificar el problema de regulación general: Si existen diferentes nodos desviados en el SDL y en el STR en diferentes áreas, el problema es general.
Bloque 4: La función de este bloque es determinar los recursos con los cuales se van a corregir las desviaciones en los nodos de referencia en el SDL, de acuerdo con el tipo de problema identificado en el bloque anterior. La determinación de estos recursos se realiza de acuerdo a nuevas reglas heurísticas:

Regla heurística para resolver el problema de regulación local: La desviación de tensión del nodo de la zona i, se corrige utilizando su condensador (si existe en este nodo) y el tap del transformador ubicado en este nodo de referencia del SDL afectado. Las maniobras se realizan de acuerdo al tipo de desviación: si este nodo presenta una sobretensión el condensador debe desconectarse (si existe), si la sobretensión persiste debe modificarse el tap del transformador ubicado en este nodo afectado. En el caso que este nodo presente una subtensión debe modificarse primero la posición del tap del transformador ubicado en este nodo afectado y sí el problema persiste, debe conectarse el condensador (si existe) de este nodo.

Regla heurística para resolver el problema de regulación de zona: La desviación de tensión de los nodos de la zona i, se corrige variando los recursos de reactivos de la misma zona: reactivos de cogeneradores y generadores, y los taps de los transformadores del nodo de referencia del STR asociado a la zona. Estas maniobras pueden realizarse a través del SCADA de EPSA. También existen unos condensadores que están ubicados en los nodos del SIN cercanos a la zona afectada, y en caso de requerirse maniobras sobre estos equipos se coordinan vía telefónica con el Centro Nacional de Despacho (CND).

Las maniobras se realizan de acuerdo al tipo de desviación: si los nodos de la zona presentan sobretensión primero se disminuye potencia reactiva de los cogeneradores y generadores hasta llevarlos al límite mínimo, luego si el problema persiste se desconectan los condensadores del SIN (uno a uno), finalmente si el problema continua se modifica la posición de los taps del transformador del nodo de referencia del STR asociados a la zona. En el caso que los nodos de la zona presenten una 
Tabla 2. Casos de validación realizados al SE

\begin{tabular}{|c|c|c|c|c|c|c|}
\hline & Zona & Nodo Z1 & Nodo Z2 & Nodo Z3 & Nodo Z4 & Nodo Z5 \\
\hline \multirow{3}{*}{ Local } & Sobretensión & $O K$ & $O K$ & $O K$ & $O K$ & $O K$ \\
\hline & Subtensión & $O K$ & $O K$ & $O K$ & $O K$ & $O K$ \\
\hline & Zona & Nodo Z1 & Nodo Z2 & Nodo Z3 & Nodo Z4 & Nodo Z5 \\
\hline \multirow[t]{3}{*}{ Zonal } & Sobretensión & $O K$ & $O K$ & $O K$ & $O K$ & $O K$ \\
\hline & Subtensión & $O K$ & $O K$ & $O K$ & $O K$ & $O K$ \\
\hline & Área & $A 1(Z 1-Z 2)$ & $A 2(Z 3-Z 4)$ & $A 3(\mathrm{Z5})$ & & \\
\hline \multirow[t]{3}{*}{ Área } & Sobretensión & $O K$ & $O K$ & $O K$ & & \\
\hline & Subtensión & $O K$ & $O K$ & $O K$ & & \\
\hline & Área & $A 1 A 2$ & $A 1 A 3$ & $A 2 A 3$ & $A 1 A 2 A 3$ & \\
\hline \multirow[t]{2}{*}{ General } & Sobretensión & $O K$ & $O K$ & $O K$ & $O K$ & \\
\hline & Subtensión & $O K$ & $O K$ & $O K$ & $O K$ & \\
\hline
\end{tabular}

subtensión debe modificarse primero la posición del tap del transformador del nodo del STR de la zona, luego si el problema persiste deben conectarse los condensadores del SIN (uno a uno) y por último si el problema continua se inyectan reactivos desde los cogeneradores y generadores hasta llevarlos al límite máximo.

Regla heurística para resolver el problema de regulación de área: La desviación de tensión de los nodos del área i, se corrige variando los recursos de reactivos de la misma área: reactivos de cogeneradores y generadores y los taps de los transformadores ubicados en todos los nodos de referencia del STR asociados al área. Estas maniobras pueden realizarse a través del SCADA de EPSA. También existen unos condensadores y reactores que están ubicados en los nodos del SIN cercanos al área afectada, y en caso de requerirse maniobras sobre estos equipos se coordinan vía telefónica con el CND.

Las maniobras se realizan de acuerdo al tipo de desviación: si los nodos del área presentan sobretensión, primero se disminuye potencia reactiva de los cogeneradores y generadores hasta llevarlos al límite mínimo, luego si el problema persiste se modifica la posición de los taps de los transformadores de todos los nodos del STR asociados al área, si no hay mejoras se desconectan los condensadores del SIN (uno a uno), y finalmente se deben conectar los reactores del SIN asociados a cada área. En el caso en que el nodo presente una subtensión se deben ejecutar las acciones sobre los elementos, al contrario de cuando se presentaba una sobretensión.

Regla heurística para resolver el problema de regulación general: La desviación de tensión de los nodos de referencia de diferentes áreas, se corrige variando los recursos de reactivos en las áreas afectadas: reactivos de cogeneradores y generadores y los taps de los transformadores ubicados en los todos los nodos de referencia del STR asociados a las área afectadas. Estas maniobras pueden realizarse a través del SCADA de EPSA. También existen unos condensadores y reactores que están ubicados en los nodos del SIN cercanos a las áreas afectadas y la línea de $500 \mathrm{KV}$, en caso de requerirse maniobras sobre estos equipos se coordinan vía telefónica con el CND.

Las maniobras se realizan de acuerdo al tipo de desviación: si todos los nodos desviados de las áreas presentan sobretensión primero se disminuye potencia reactiva de los cogeneradores y generadores hasta llevarlos al límite mínimo, si el problema persiste se modifica la posición de los taps de los transformadores de todos los nodos del STR asociados a las áreas afectadas, luego si el problema no se resuelva se desconectan los condensadores del SIN (uno a uno) cercanos a todas las áreas afectadas, si el problema aún continua se deben conectar los reactores asociados a cada área y finalmente se debe abrir la línea de 
$500 \mathrm{KV}$. En el caso en que el nodo presente una subtensión se deben ejecutar las acciones sobre los elementos, al contrario de cuando se presentaba una sobretensión.

\subsubsection{Señales de salida del SE}

Las señales de salida del sistema experto se clasifican en:

Listado de nodos de referencia del SDL y STR por zonas y por áreas.

Tipo de problema de regulación de tensión en la zona y en el área.

Mensajes para el operador para comunicarse con el CND para que realicen las maniobras sobre los recurso del SIN

Listado de maniobras que se deben ejecutar sobre los recursos de potencia reactiva, con el fin de corregir las desviaciones de tensión para cada tipo de problema.

\subsection{Validación del SE}

El SE se desarrolló en el lenguaje de programación de la herramienta computacional Matlab, su validación se realizó de la sgte forma:

Se validaron todas las Reglas Heurísticas que permitían identificar el tipo de problema de regulación de tensión, en la Tabla 2 se presentan todos los casos de validación realizados en el SEP en estudio.

Una vez identificado el tipo de problema de regulación de tensión, se validaron las maniobras encaminadas a resolver las desviaciones de acuerdo a las Reglas Heurísticas correspondientes.

Los casos utilizados en la validación, fueron seleccionados de la base de datos de los históricos. Para cada uno de estos casos se completó la información de las señales de entrada requeridas en el SE. Estas condiciones iniciales de operación real del SEP, se introducían en el SEP simulado en el Digsilent, sus salidas de las tensiones en diferentes nodos de referencia verificaban que para estas condiciones de operación se presentaban sobretensiones ó subtensiones. Estas salidas del Digsilent se utilizaron como señales de entrada al SE, el cual las procesaba y entregaba sus señales de salida indicando que maniobras debían ejecutarse sobre los recursos de reactivos del SEP modelado, al ejecutar estas maniobras los nodos desviados mejoraban su desviación. El ciclo se repetía hasta que no se presentó ningún nodo con desviación de tensión. En la Figura 4 se ilustra el esquema de validación utilizado.

\section{Resultados y discusión}

En el SE se han probado todos los tipos de problema de regulación de tensión alta y baja para el sistema

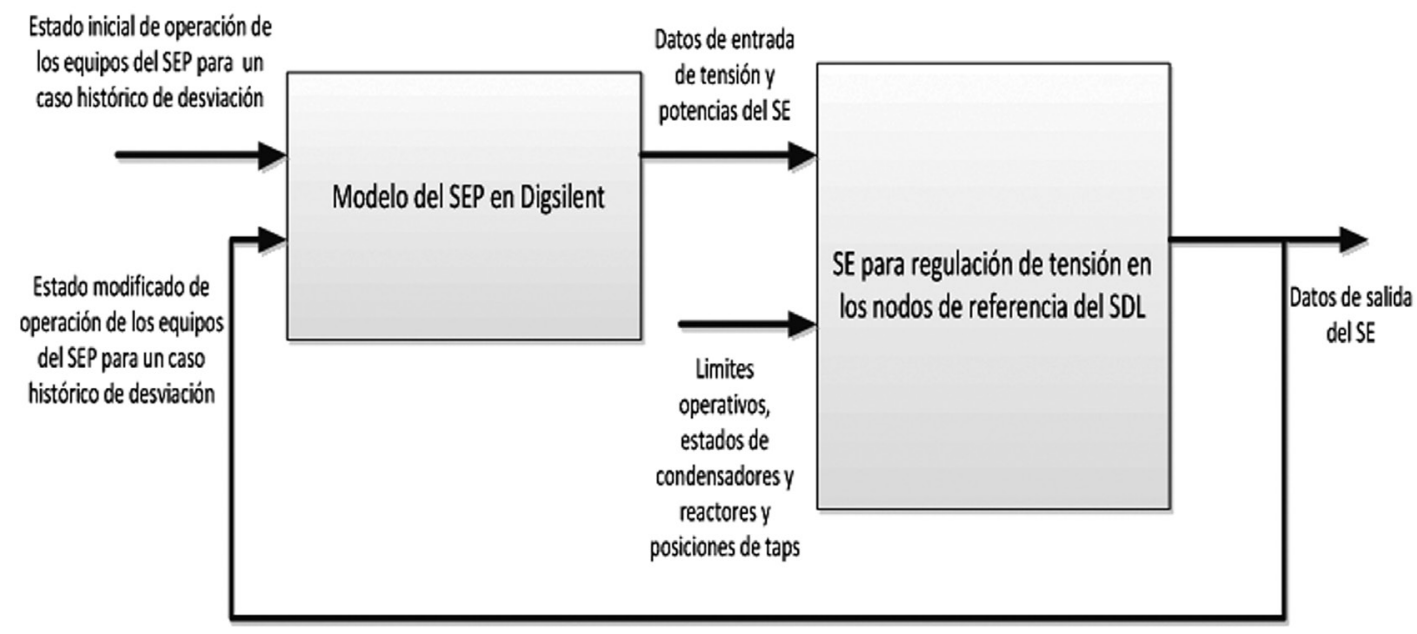

Figura 4. Estructura de validación del SE propuesto para regular tensión 
Tabla 3. Resultados validación del SE para un problema tipo general de sobretensión

\begin{tabular}{|c|c|c|c|c|c|}
\hline NODOS & $\begin{array}{c}\text { VNOMINAL } \\
(K V)\end{array}$ & $\begin{array}{c}V M I N \\
(K V)\end{array}$ & $\begin{array}{c}\boldsymbol{V} \boldsymbol{M A X} \\
(\boldsymbol{K} V)\end{array}$ & $\begin{array}{c}V \\
\text { PREVIO } \\
(K V)\end{array}$ & $\begin{array}{c}V \\
\text { POSTERIOR } \\
(K V)\end{array}$ \\
\hline V1 (San marcos $220 \mathrm{KV})$ & 230 & - & - & 230 & 230 \\
\hline Vla (Cartago $115 \mathrm{KV})$ & 115 & 117 & 121 & 122,32 & 120,05 \\
\hline V1b (Calima $115 \mathrm{KV})$ & 115 & 113 & 121 & 121,15 & 117,88 \\
\hline V1c (San Marcos $115 \mathrm{KV})$ & 115 & 116 & 121 & 123,31 & 119,84 \\
\hline V1d (Bajo Anchicayá 115 KV) & 115 & 117 & 121 & 121,17 & 117,54 \\
\hline Vle (Buga $115 \mathrm{KV})$ & 115 & 113 & 120 & 121,12 & 117,85 \\
\hline V1f (Sta. Bárbara $115 \mathrm{KV})$ & 115 & 113 & 120 & 122,66 & 119,17 \\
\hline V1g (Pance $115 \mathrm{KV})$ & 115 & 115 & 121 & 118,77 & 115,25 \\
\hline V2 (Cartago 34,5 KV) & 34,5 & 34,5 & 35,5 & 36,40 & 35,71 \\
\hline V3 (Cartago 13,2 KV) & 13,2 & 12,9 & 13,9 & 14,08 & 13,82 \\
\hline V4 (La Unión 34,5 KV) & 34,5 & 34 & 36 & 36,40 & 35,60 \\
\hline V5 (Zarzal 34,5 KV) & 34,5 & 34 & 36 & 36,38 & 35,55 \\
\hline V6 (Tuluá 34,5 KV) & 34,5 & 34 & 36 & 35,08 & 35,08 \\
\hline$V 7$ (Buga 34,5 KV) & 34,5 & 34,5 & 35,8 & 37,41 & 36,40 \\
\hline V8 (Calima 34,5 KV) & 34,5 & 34,5 & 36 & 37,46 & 36,44 \\
\hline V9 (Cerrito 34,5 KV) & 34,5 & 34,3 & 35,6 & 37,06 & 36,00 \\
\hline V10 (Sta. Bárbara 34,5 KV) & 34,5 & 34,7 & 35,7 & 36,70 & 35,63 \\
\hline V11 (Sta. Bárbara 13,2 KV) & 13,2 & 12,9 & 13,9 & 14,06 & 13,65 \\
\hline V12 (Candelaria $34,5 \mathrm{KV}$ ) & 34,5 & 34,4 & 35,5 & 36,34 & 35,27 \\
\hline$V 13(\operatorname{Codazzi} 13,2 \mathrm{KV})$ & 13,2 & 12,9 & 13,9 & 14,14 & 13,74 \\
\hline V14 (Jamundi 34,5 KV) & 34,5 & 34 & 35,6 & 35,53 & 34,48 \\
\hline V15 (Jamundi 13,2 KV) & 13,2 & 13,1 & 14 & 13,55 & 13,14 \\
\hline V16 (Palmaseca 34,5 KV) & 34,5 & 32,8 & 35,4 & 36,66 & 35,57 \\
\hline V17 (Palmaseca 13,2 KV) & 13,2 & 12,8 & 14 & 14,34 & 13,91 \\
\hline V18 (Termo Yumbo 34,5 KV) & 34,5 & 35 & 36,1 & 36,00 & 34,98 \\
\hline V19 (Guachal 34,5 KV) & 34,5 & 34,5 & 36 & 36,60 & 35,55 \\
\hline V20 (Juanchito 34,5 KV) & 34,5 & 34,4 & 35,8 & 36,83 & 35,74 \\
\hline V21 (Juanchito 13,2 KV) & 13,2 & 13 & 13,9 & 13,63 & 13,22 \\
\hline V22 (Meléndez 34,5 KV) & 34,5 & 34,5 & 35,8 & 36,62 & 35,53 \\
\hline V23 (Meléndez 13,2 KV) & 13,2 & 13 & 13,9 & 13,57 & 13,16 \\
\hline V24 (Chipichape 34,5 KV) & 34,5 & 34,5 & 35,5 & 36,61 & 35,52 \\
\hline V25 (Chipichape 13,2 KV) & 13,2 & 13 & 13,9 & 13,90 & 13,49 \\
\hline V26 (Tabor 13,2 KV) & 13,2 & 12,9 & 13,6 & 13,96 & 13,53 \\
\hline V27 (Pailón 13,2 KV) & 13,2 & 12,9 & 13,9 & 14,14 & 13,71 \\
\hline PORCENTAJE DE DESVIACIÓN & & & & $78,79 \%$ & $18,18 \%$ \\
\hline
\end{tabular}


de distribución de EPSA y sus resultados fueron satisfactorios. Los casos de prueba utilizados se tomaron de situaciones reales en escenarios típicos de operación: problemas de desviación de tensión locales presentados en diferentes zonas, problemas de desviación de tensión en varios nodos de una misma zona, problemas de desviación de nodos en una misma área y problemas de desviación en diferentes áreas o problema general.

Los resultados para un problema de desviación general se ilustran en la tabla 3. En la primera columna se ilustran los nodos de referencia en el SDL y STR y los nodos indicadores en el STR. Para cada uno de los nodos se tiene su valor nominal, su valor máximo y su valor mínimo de acuerdo a los rangos operativos. En la columna "previos a" se observan los valores de la tensión antes de realizar las maniobras sugeridas por el SE y en la columna "posterior a" se observan los valores de tensión después de realizar las maniobras para corregir las desviaciones. Inicialmente se tiene una desviación del tipo general, donde el 79\% de los nodos están desviados. Después de ejecutar las maniobras recomendadas por el SE, los nodos desviados se reducen al $18 \%$; para este nuevo caso se corre el SE y se identifica el nuevo tipo de problema de regulación y recomienda una nuevas maniobras que reducen el porcentaje de nodos desviados. Este procedimiento se repite hasta que el porcentaje de nodos desviado sea cero.

En la Figura 5 se pueden observar los mensaje generados por el SE que muestran el tipo de problema detectado y la secuencia de maniobras para restablecer la tensión de este problema general de alta tensión. Los resultados obtenidos para este caso fueron coherentes con lo que se planteó en las Reglas Heurísticas, las maniobras propuestas coinciden y fueron necesarias para restablecer la desviación en diferentes nodos.

\section{Conclusiones}

El SE desarrollado en esta aplicación considera el efecto individual y colectivo de los recursos de potencia reactiva con el fin de determinar las maniobras que inyecten reactivos en las zonas más cercanas a los nodos desviados, lo cual permite la regulación de tensión de una manera más efectiva. Además, permite al operador de la red responder de manera más rápida ante las desviaciones sostenidas de tensión, lo cual permite mejorar la calidad del servicio.

Los nodos de referencia del SEP deben corresponder a los nodos que cuentan con recursos de inyección de reactivos conectados o cercanos a ellos, ya que su ubicación geográfica en el SEP es clave para mantener el perfil de voltaje ante variaciones pico y normales de la carga. Este SE desarrollado se implementó en un sistema de distribución real y se validó mediante la simulación de casos presentados en diferentes escenarios del SEP. Sus resultados fueron satisfactorios para diferentes casos de desviación de altas y bajas tensiones.

Este SE es una herramienta que permite regular la tensión de manera más ágil, eficiente y oportuna frente a la regulación manual que ejecutan los operadores del Centro de Supervisión y Maniobra. Además, optimiza la cantidad de maniobras en los equipos de los nodos de distribución al evaluar el estado de los equipos de control de reactivos en el SIN y STR, contribuyendo al aumento de su vida útil.

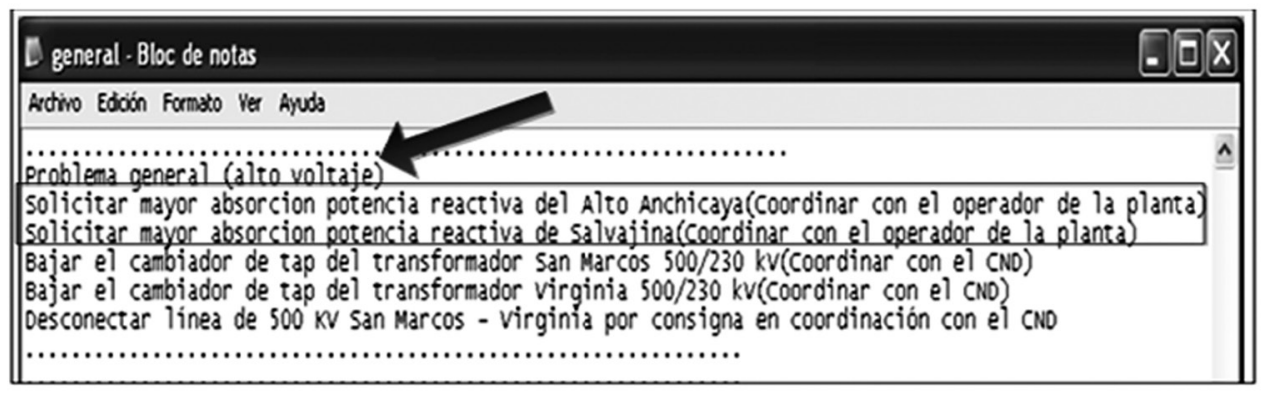

Figura 5. Estructura de validación del SE propuesto para regular tensión 


\section{Referencias bibliográficas}

A. Azmy, (2007). Optimal Power Flow to Manage Voltage Profiles in Interconnected Networks Using Expert Systems. IEEE transactions on power systems, vol. 22, no. 4.

Gehao, S. Xiuceng, J. \& Yi, Z. (2005), Optimal Coordination For Multi-Agent Based Secondary Voltage Control In Power System Transmission and Distribution. Conference \& Exhibition: Asia and Pacific Dalian, China.
P. Kundur, (1994), Power System stability and control, New York, Mc Graw-Hill

Ruey-Hsun Liang, (2003). Fuzzy Based Reactive Power And Voltage Control in a Distribution System. IEEE, and Yung-Shuen Wang.

Vaschetti, J.C., \& Sauchelli, V. H. (2012). Control Automático de Voltaje en Sistemas Eléctricos. Información Tecnológica, Cordoba, Argentina. Información Tecnológica Vol. 23 $\mathrm{N}^{\circ} 52012$.

Revista Ingeniería y Competitividad por Universidad del Valle se encuentra bajo una licencia Creative Commons Reconocimiento - Debe reconocer adecuadamente la autoría, proporcionar un enlace a la licencia e indicar si se han realizado cambios. Puede hacerlo de cualquier manera razonable, pero no de una manera que sugiera que tiene el apoyo del licenciador o lo recibe por el uso que hace. 